\title{
Thermodynamics of an interacting trapped Bose-Einstein gas in the classical field approximation
}

\author{
Krzysztof Góral ${ }^{1}$, Mariusz Gajda ${ }^{2}$, and Kazimierz Rzążewski ${ }^{1}$ \\ ${ }^{1}$ Center for Theoretical Physics, ${ }^{2}$ Institute of Physics, \\ Polish Academy of Sciences, Aleja Lotników 32/46, 02-668 Warsaw, Poland
}

\begin{abstract}
We present a convenient technique describing the condensate in dynamical equilibrium with the thermal cloud, at temperatures close to the critical one. We show that the whole isolated system may be viewed as a single classical field undergoing nonlinear dynamics leading to a steady state. In our procedure it is the observation process and the finite detection time that allow for splitting the system into the condensate and the thermal cloud.
\end{abstract}

PACS numbers: 03.75.Fi

Successful experimental realization of Bose-Einstein condensation in a dilute gas of alkali atoms [1] offers a new and fascinating tool to probe the border between quantum and classical worlds. The theory of the dynamical behavior of such a many body system is very difficult and cannot be solved exactly. It is particularly hard to extract quantitative predictions in the vicinity of the critical temperature. At the other extreme, at zero temperature, the weakly interacting Bose gas is well described by the mean-field approach. All particles occupy the same quantum state whose wave function is the lowest energy solution of the Gross-Pitaevskii (GP) equation [2]. At low temperatures the Bogoliubov approximation comes handy [3] with its quasi-particles that have just been observed in a direct experiment [ 4 ]. The theory gets much more complex at higher temperatures. There is a considerable effort to develop a working theoretical and numerical tool valid there. One group of papers [5, 6, 7, 8, 9, from the very beginning describes the system as consisting of two (interacting) fractions: the condensate and the thermal cloud. This ambitious program is very demanding numerically. The other group interprets the high energy solutions of the time-dependent GP equation as describing the full condensate plus thermal cloud system [10]. As a rule, these authors were able to identify the condensate as a definite part of the system only in the somewhat academic case of the gas in a rectangular box with periodic boundary conditions. In this case the condensate is just the zero momentum component of the wave function. The aim of this Rapid Communication is to show that much more may be achieved with the readily available high-energy solutions of the GP equation in harmonic traps. It is also possible to split this solution into a sum of the condensed and uncondensed parts, define the condensate wave function and approximately estimate the temperature of the resulting system.

The Hamiltonian $\mathrm{H}$ of the system takes the form:

$$
\begin{aligned}
H= & \int \mathrm{d}^{3} r \hat{\Psi}^{\dagger}(\mathbf{r}, t)\left(\frac{p^{2}}{2 m}+\frac{1}{2} m \omega^{2} r^{2}\right) \hat{\Psi}(\mathbf{r}, t) \\
& +\frac{2 \pi \hbar^{2} a_{s}}{m} \int \mathrm{d}^{3} r \hat{\Psi}^{\dagger}(\mathbf{r}, t) \hat{\Psi}^{\dagger}(\mathbf{r}, t) \hat{\Psi}(\mathbf{r}, t) \hat{\Psi}(\mathbf{r}, t),
\end{aligned}
$$

where $\hat{\Psi}(\mathbf{r}, t)$ is a field operator that destroys a particle at position $\mathbf{r}$ and obeys standard bosonic commutation relations
$\left[\hat{\Psi}(\mathbf{r}, t), \hat{\Psi}^{\dagger}\left(\mathbf{r}^{\prime}, t\right)\right]=\delta\left(\mathbf{r}-\mathbf{r}^{\prime}\right)$. The first term describes particles with mass $m$ trapped in a potential of a spherically symmetric harmonic oscillator of frequency $\omega$ while the second term describes two-body interactions. Here we have assumed that particles interact via a contact potential $V\left(\mathbf{r}-\mathbf{r}^{\prime}\right)=$ $4 \pi \hbar^{2} a_{s} \delta\left(\mathbf{r}-\mathbf{r}^{\prime}\right) / m$, where $a_{s}$ is the s-wave scattering length. The Heisenberg equation originating from this Hamiltonian acquires the following form

$$
\begin{aligned}
i \hbar \frac{\partial \hat{\Psi}(\mathbf{r}, t)}{\partial t}= & \left(-\frac{\hbar^{2} \nabla^{2}}{2 m}+\frac{1}{2} m \omega^{2} r^{2}\right) \hat{\Psi}(\mathbf{r}, t) \\
& +\frac{4 \pi \hbar^{2} a_{s}}{m} \hat{\Psi}^{\dagger}(\mathbf{r}, t) \hat{\Psi}(\mathbf{r}, t) \hat{\Psi}(\mathbf{r}, t) .
\end{aligned}
$$

A full operator solution of the non-linear Eq.(2) is not known. In addition, the identification of a condensate phase is also a subtle issue. The only exception is a system of particles in a periodic box. Here the symmetry of the problem helps: natural eigenmodes of the system are plane waves with a quantized momentum $\mathbf{k}=2 \pi\left(j_{1}, j_{2}, j_{3}\right) / L$ ( $j_{i}$ being integer, $L$ - box size). Therefore, the field operator can be expanded in these modes:

$$
\hat{\Psi}(\mathbf{r}, t)=\frac{1}{L^{3 / 2}} \sum_{\mathbf{k}} \exp (-i \mathbf{k} \cdot \mathbf{r}) \hat{a}_{\mathbf{k}}(t) .
$$

A Bose-Einstein condensate can be uniquely associated with the zero momentum mode, $\mathbf{k}=0$. The annihilation and creation operators $\hat{a}_{\mathbf{k}}, \hat{a}_{\mathbf{k}}^{\dagger}$ satisfy non-linear equations following from Eq.(2).

Different approximate approaches mentioned above 15,6 . 7. 8, 9] have been introduced in order to solve the problem. Here we want to utilize a method which has been extensively and successfully explored in quantum optics. A quantum field operator describing a coherent electromagnetic field (such as laser light) can be replaced by a complex valued classical field. Such a substitution is justified for these modes that are highly occupied. Only then are quantum fluctuations negligible and the non-vanishing commutator may be ignored. This kind of approximation has been used recently in [10] for the matter field: $\hat{a}_{\mathbf{k}} \rightarrow a_{\mathbf{k}}$. Note that $\sum_{\mathbf{k}}\left|a_{\mathbf{k}}\right|^{2}=N$ and the condensate occupation is equal to $n_{0}=\left|a_{0}\right|^{2}$. On the other hand, it follows from Eqs.(2)-(3) that the semi-classical approximation is equivalent to a substitution: $\hat{\Psi}(\mathbf{r}, t) \longrightarrow \sqrt{N} \Psi(\mathbf{r}, t)=$ 
$\frac{1}{L^{3 / 2}} \sum_{\mathbf{k}} a_{\mathbf{k}} \exp (-i \mathbf{k} \cdot \mathbf{r})$, where $\Psi(\mathbf{r}, t)$ fulfills the standard GP equation. According to conventional wisdom, this equation describes a pure condensate at zero temperature, hence the whole classical field $\Psi(\mathbf{r}, t)$ represents the condensate populated by $n_{0}=N$ particles [11, 12].

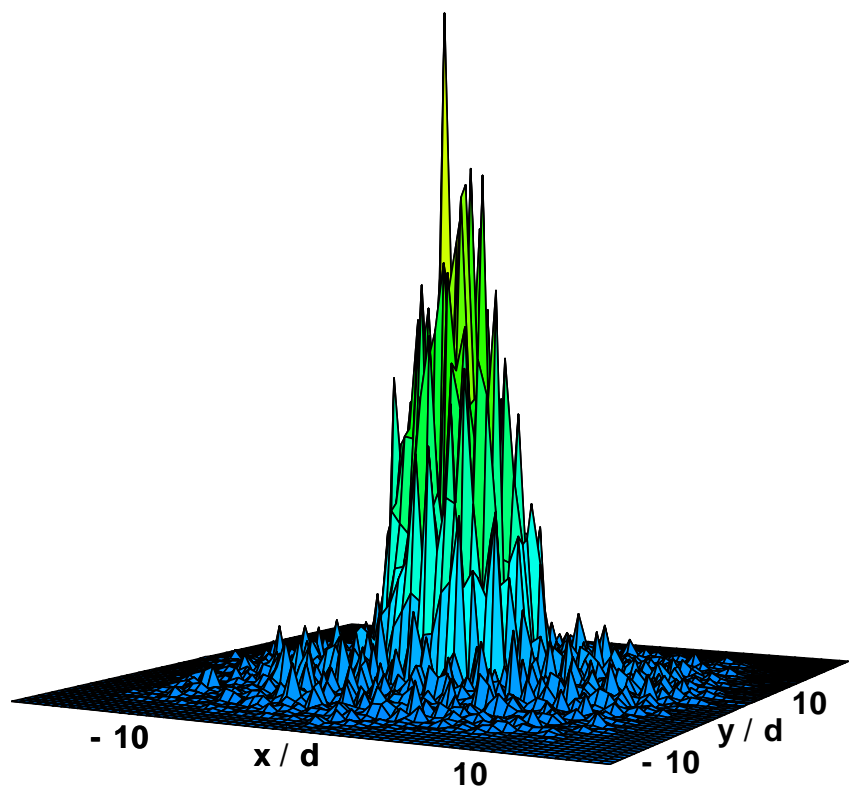

FIG. 1: Cross-section of the instantaneous snapshot of the density distribution in the $z=0$ plane of the Bose gas with the total energy $E=70 \hbar \omega$. In this and all the following figures lengths are given in units of $d=\sqrt{\hbar / m \omega}$.

It seems that the two contradicting interpretations of the high-energy solution coexist in the literature. In the following we will clarify this seeming incongruity. We focus our attention on experimentally relevant system of $100000{ }^{87} \mathrm{Rb}$ atoms trapped in the spherically symmetric harmonic potential of frequency $\omega=2 \pi 100 \mathrm{~Hz}$. Our procedure is as follows. First, we define the wave function of the system on a spatial three-dimensional grid. Initial values of the wave function at each point are chosen in accordance with the constraints of a fixed energy and particle number. Next, we propagate this state in time, solving the time dependent GP equation with the help of the Fast Fourier Transform. It occurs that for the given total energy and particle number the same steady state is reached after several milliseconds. We have checked that the steady state attained does not depend on the choice of the initial wave function (in particular, on the fact whether its phase is random or not) as long as the number of particles and the total energy are kept constant.

The high-energy solutions of the GP equation have striking features [11]. A snapshot of the density distribution is shown in Fig.1. The density is extremely irregular and exhibits a number of sharp spikes changing its shapes and positions on very short time scales. Typical methods used to monitor trapped atomic condensates involve optical techniques. A condensate is illuminated by laser light which, after passing through the atomic system (and some optical ele- ments), is monitored by a CCD camera. The exposure time $\Delta t$ varies from a few microseconds to hundreds of milliseconds and within this time the condensate density undergoes rapid changes due to the fast dynamics but also because of quantum fluctuations. The nature of the observation process introduces a kind of smearing. In fact, in such a long exposure a timeaveraged rather then an instantaneous single-particle density is monitored. The existence of short time scales in non-linear many-body dynamics has to be taken into account. The object of physical significance is therefore a time-averaged singleparticle density matrix:

$$
\overline{\rho\left(\mathbf{r}_{1}, \mathbf{r}_{2}, t\right)}=\frac{1}{\Delta t} \int_{t-\Delta t / 2}^{t+\Delta t / 2} \Psi^{*}\left(\mathbf{r}_{1}, t^{\prime}\right) \Psi\left(\mathbf{r}_{2}, t^{\prime}\right) d t^{\prime} .
$$

This coarse graining procedure destroys the purity of a state of the system. After the time averaging all irregularities of the density are being smoothed out especially in the inner part of the density profile (see Fig,2). The resemblance to the well publicized photographs of the experimental condensates at intermediate temperatures is striking [13]. Varying the energy of the gas we can scan the whole range from the pure condensate all the way to a nearly critical condition of no condensate. The sample profiles of the time-averaged column density (integrated along the z-axis) are shown in Fig.2. In parts A and B of Fig.2 note the bimodal structure of the distribution: the central peak corresponding to the Bose-Einstein condensate and the broad background identified with the thermal cloud.

We can provide a quantitative analysis of the resulting averaged state of the Bose gas. To this end we recall a classical definition of the condensate by means of the spectral decomposition of the single-particle density matrix [14]. Diagonalization of a single-particle time-averaged density matrix leads to natural eigenmodes $\psi_{i}(\mathbf{r}, t)$ and eigenvalues $n_{i} / N$ :

$$
\overline{\rho\left(\mathbf{r}_{1}, \mathbf{r}_{2}, t\right)}=\sum_{i} \frac{n_{i}}{N} \psi_{i}^{\star}\left(\mathbf{r}_{1}, t\right) \psi_{i}\left(\mathbf{r}_{2}, t\right)
$$

The system may be viewed as a mixture of many coupled coherent modes $\psi_{i}$ whose occupation is $n_{i}$. The self-consistency of the model requires that population of each single mode $\psi_{i}$ is large, $n_{i}>1$. Otherwise the substitution of the field operator $\hat{\Psi}(\mathbf{r}, t)$ by a wave function $\Psi(\mathbf{r}, t)$ would not be justified. The existence of the dominant eigenvalue $n_{0} / N$ of the order of unity signifies the presence of the condensate with the corresponding eigenvector $\psi_{0}$ being its wave function. As the modes and their occupation are known only after the time averaging, the verification of the semi-classical criterion can only be done a posteriori. We try a number of grids and choose the one that yields the highest occupation of all modes, justifying the semi-classical approximation.

The time averaging followed by a diagonalization of the density matrix gives the spatial modes of the system. These modes are typically not known even in a stationary case. Still, the only exception is the box with periodic boundary conditions. Let us illustrate our method using again this simple case. 


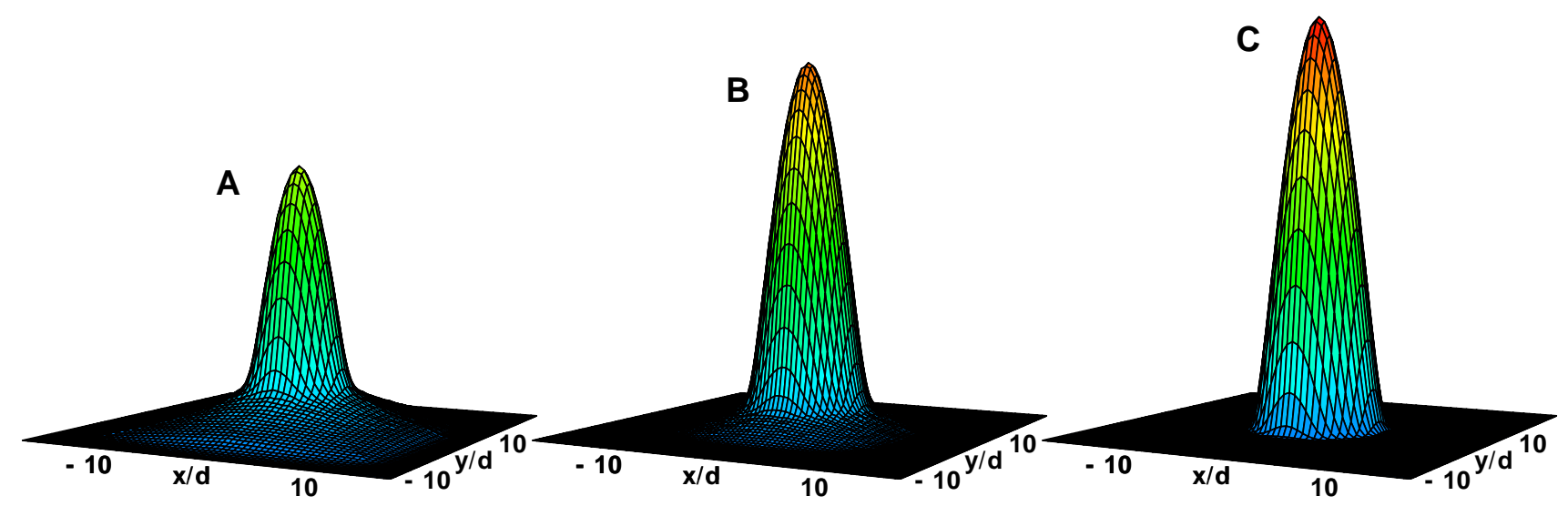

FIG. 2: Time-averaged stationary column density distribution for a Bose gas plotted for different values of total energy $E$. A $E=$ $70.00 \hbar \omega$ (30\% atoms in condensate). B $E=23.67 \hbar \omega$ ( $72 \%$ of atoms in condensate). $\mathbf{C} E=13.04 \hbar \omega$ (pure condensate).

Substitution of all annihilation operators by complex amplitudes $\hat{a}_{\mathbf{k}} \rightarrow a_{\mathbf{k}}$ gives the following expression for the singleparticle density matrix:

$$
\overline{\rho\left(\mathbf{r}_{1}, \mathbf{r}_{2}, t\right)} \approx \frac{1}{V} \sum_{\mathbf{k}} \exp \left[i \mathbf{k} \cdot\left(\mathbf{r}_{1}-\mathbf{r}_{2}\right)\right]\left|a_{\mathbf{k}}(t)\right|^{2}
$$

and the time averaging leaves only diagonal elements $\mathbf{k}=\mathbf{k}^{\prime}$ as the off-diagonal ones oscillate rapidly and get rapidly dephased, $\overline{a(t)_{\mathbf{k}}^{*} a(t)_{\mathbf{k}^{\prime}}} \approx \delta_{\mathbf{k}, \mathbf{k}^{\prime}}\left|a_{\mathbf{k}}(t)\right|^{2}$. Obviously, the $\left|a_{\mathbf{k}}\right|^{2}$ are populations of different modes. Only if one introduces a kind of coarse graining leading to a suppression of the off-diagonal elements of the single-particle density matrix can one identify the $\mathbf{k}=0$ mode as a Bose-Einstein condensate with an occupation given by $\left|a_{0}\right|^{2}$. Without this additional assumption the whole complex field $\Psi(\mathbf{r}, t)$ describes one coherent, dynamically evolving Bose-Einstein condensate without any thermal cloud. This kind of interpretation is used in [11, 12]. On the contrary, identification of the $\mathbf{k}=0$ momentum component of $\Psi(\mathbf{r}, t)$ with a Bose condensate has been directly assumed in [10]. Our analysis solves this apparent contradiction. By examining a detection process and the relevant time scale we can uniquely determine the condensate fraction, its wave function as well as the structure of excited modes of the interacting system. Moreover, the method is no longer limited to an academic problem of the uniform system. A steady-state singleparticle density matrix for the spherically symmetric trapping potential must have eigenvectors that are simultaneously diagonalizing the angular momentum operators. Therefore, the eigenvectors must be proportional to the spherical harmonics $Y_{l m}$. What remains is the one dimensional diagonalization in the radial variable of the following projection of the density matrix:

$$
\rho_{l m}\left(r, r^{\prime}\right)=\int \overline{\rho\left(r, \Omega ; r^{\prime}, \Omega^{\prime}\right)} Y_{l m}^{\star}(\Omega) Y_{l m}\left(\Omega^{\prime}\right) d \Omega d \Omega^{\prime},
$$

where the integration is performed over solid angles $\Omega$ and $\Omega^{\prime}$ associated with the corresponding particle coordinates. We expect the condensate to be present in the zero angular momentum component of the single-particle density matrix. Indeed, in the inset of Fig. 3 we show a typical distribution of the eigenvalues of the $l=0$ part of the density matrix with one dominant eigenvalue and with the corresponding eigenfunction plotted in Fig. 3 together with the whole time-averaged radial density distribution.

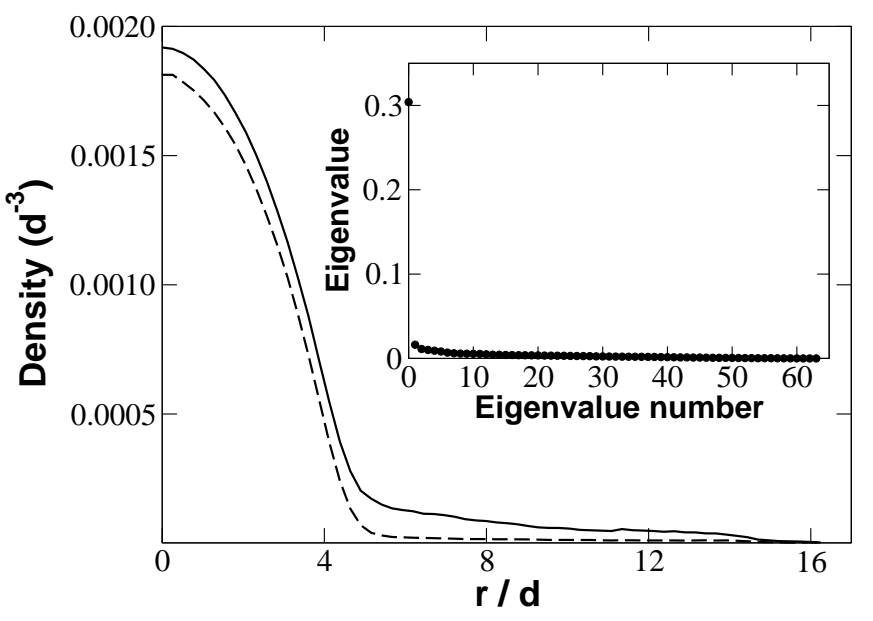

FIG. 3: Time-averaged radial density profile of a stationary state of a Bose gas. The total energy is $E=70.00 \hbar \omega$. Density of the whole system - solid line; profile of the Bose-Einstein condensate (defined as a dominant eigenmode of $\left.\rho_{00}\left(r_{1}, r_{2}\right)\right)$ - dashed line. All eigenvalues of $\rho_{00}\left(r_{1}, r_{2}\right)$ are shown in the inset.

The last point raised here is the question of temperature. In principle we could do something similar to a typical experiment: switch off the trapping potential, let the gas expand and analyze the properties of the thermal part. Numerical limitations and the constraining condition of the high occupation of each eigenmode make it hard. Alternatively, we can follow the procedure of [15] and fit the profile of the thermal fraction of the ideal Bose gas to the outer part of our averaged density. 
This yields a reasonable, order of magnitude result. The temperature dependence of the condensed fraction together with its large uncertainty is shown in Fig. 4 . In the inset we plotted the energy dependence of the number of atoms in the condensate. We compare our results with the ideal gas and with a two-gas estimate (finite-temperature Hartree-Fock scheme [16]). We see a reasonably good agreement between all three calculations.

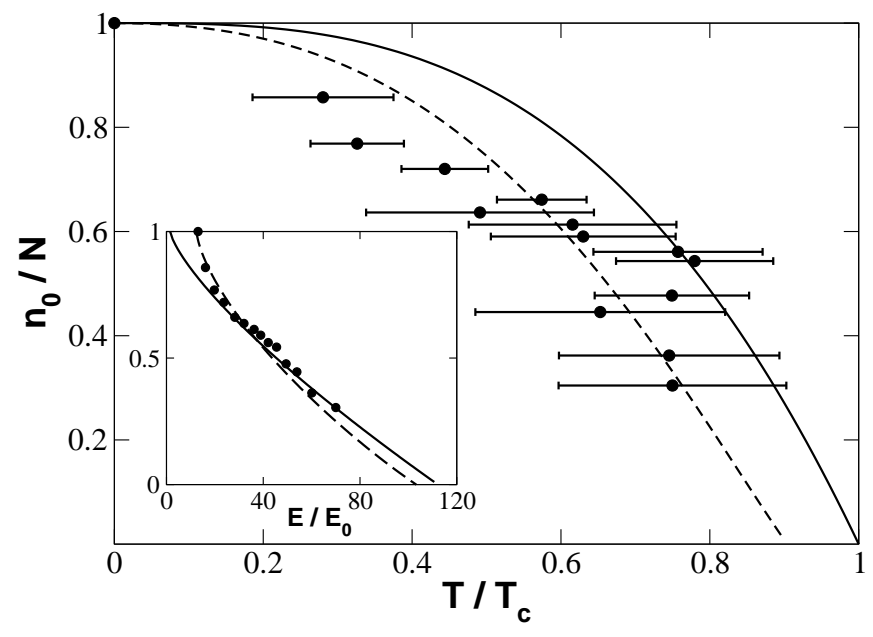

FIG. 4: Condensate fraction versus temperature or (the inset) the total energy of the system. The solid line represents the ideal gas in the thermodynamic limit, the dashed line depicts the results of [16] obtained within a finite-temperature Hartree-Fock scheme, while dots show our data. Error bars indicate a range of temperatures for which we obtain acceptable fits of the ideal Bose gas distribution to the outer wings of the thermal cloud. $T_{c}$ is the ideal gas critical temperature in the thermodynamic limit and $E_{0}=\hbar \omega$.

To summarize: we have shown a simple way of numerically simulating the stationary dynamics of a weakly interacting trapped Bose gas at finite temperature, which uses a semi-classical representation of the matter field and, in accordance with the experimental procedures, stresses the role of a finite exposure time when photographing the condensate. In the self-consistent determination of the condensate fraction we rely solely on the classic criterion of Onsager and Penrose. This way we avoid an arbitrary splitting of the system into the condensed and thermal components from the very beginning. The method may be used to model the impact of thermal fluctuations on the dynamical processes with the condensate, such as solitons or vortices. The next step in going beyond the approximations employed in our model is the estimation of the influence of quantum corrections to the classical fields, in particular the study of the corresponding time scales.

We thank J. Mostowski and G.V. Shlyapnikov for stimulating discussions. K.R. is supported by the subsidy of the
Foundation for Polish Science. K.G. and M.G. acknowledge support by Polish KBN grants 5 P03B 10220 and 2 PO3B 078 19 , respectively. Numerical calculations have been performed using computers at Interdisciplinary Centre for Mathematical and Computational Modelling of Warsaw University.

[1] M.H. Anderson et al., Science 269, 198 (1995); K.B. Davis et al., Phys. Rev. Lett. 75, 3969 (1995); C.C. Bradley et al., ibid. 75, 1687 (1995); 79, 1170(E) (1997).

[2] L.P. Pitaevskii, Zh. Eksp. Teor. Fiz. 40, 646 (1961) [Sov. Phys. JETP 13, 451 (1961)]; E.P. Gross, Nuovo Cimento 20, 454 (1961).

[3] N. Bogoliubov, J. Phys. (Moscow) 11, 23 (1947).

[4] J.M. Vogels et al., Phys. Rev. Lett. 88, 060402 (2002).

[5] P.O. Fedichev, G.V. Shlyapnikov, and J.T.M. Walraven, Phys. Rev. Lett. 80, 2269 (1998); H. Shi and A. Griffin, Phys. Rep. 304, 1 (1998); S. Giorgini, Phys. Rev. A 61, 063615 (2000).

[6] D.A.W. Hutchinson, E. Zaremba, and A. Griffin, Phys. Rev. Lett. 78, 1842 (1997); S. Giorgini, L. P. Pitaevskii, and S. Stringari, ibid. 78, 3987 (1997); R. J. Dodd et al., Phys. Rev. A 57, R32 (1998); S. A. Morgan, J. Phys. B 33, 3847 (2000).

[7] N.P. Proukakis, K. Burnett, and H.T.C. Stoof, Phys. Rev. A 57, 1230 (1998); R. Walser et al., Phys. Rev. A 59, 3878 (1999).

[8] A. Sinatra, C. Lobo, and Y. Castin, Phys. Rev. Lett. 87, 210404 (2001); R.A. Duine and H.T.C. Stoof, Phys. Rev. A 65, 013603 (2002).

[9] R.J. Dodd, M. Edwards, and C.W. Clark, J. Phys. B 32, 4107 (1999); U. Al Khawaja and H.T.C. Stoof, Phys. Rev. A 62, 053602 (2000); R. Bach, M. Brewczyk, and K. Rzążewski, J. Phys. B 34, 3575 (2001); B. Jackson and E. Zaremba, Phys. Rev. Lett. 87, 100404 (2001).

[10] B.V. Svistunov, J. Mosc. Phys. Soc. 1, 373 (1991); K. Damle, S.N. Majumdar, and S. Sachdev, Phys. Rev. A 54, 5037 (1996); Yu. Kagan and B.V. Svistunov, Phys. Rev. Lett. 79, 3331 (1997); K. Góral, M. Gajda, and K. Rza̧żewski, Opt. Express 8, 92 (2001); M. J. Davis, S.A. Morgan, and K. Burnett, Phys. Rev. Lett. 87, 160402 (2001); M.J. Davis, R.J. Ballagh, and K. Burnett, J. Phys. B 34, 4487 (2001); N.G. Berloff and B.V. Svistunov, Phys. Rev. A 66, 013603 (2002) ; K.Staliunas, condmat/0110258.

[11] R.J. Marshall et al., Phys. Rev. A 59, 2085 (1999).

[12] P. Villain and M. Lewenstein, Phys. Rev. A 62, 043601 (2000); M. Leadbeater et al., Phys. Rev. Lett. 86, 1410 (2001).

[13] D. S. Durfee and W. Ketterle, Opt. Express 2, 299 (1998).

[14] O. Penrose, Philos. Mag. 42, 1373 (1951); O. Penrose and L. Onsager, Phys. Rev. 104, 576 (1956); C.N. Yang, Rev. Mod. Phys. 34, 694 (1962).

[15] for details see W. Ketterle, D.S. Durfee, and D.M. StamperKurn, in Bose-Einstein Condensation in Atomic Gases, Proceedings of the International School of Physics "Enrico Fermi", Course CXL, edited by M. Inguscio, S. Stringari, and C.E. Wieman (IOS Press, Amsterdam, 1999).

[16] see Sec. V.C of F. Dalfovo, S. Giorgini, L.P. Pitaevskii, and S. Stringari, Rev. Mod. Phys. 71, 463 (1999). 